LA-UR- $\quad 24=325$

Title: A Two-Timescale Approach to Nonlinear Model Predictive Control

Authors: Kevin L. Buescher and Christopher C. Baum

\title{
DISCLAIMER
}

Submitted to:

This report was prepared as an account of work sponsored by an agency of the United States Government. Neither the United States Government nor any agency thereof, nor any of their employees, makes any warranty, express or implied, or assumes any legal liability or responsibility for the accuracy, completeness, or usefulness of any information, apparatus, product, or process disclosed, or represents that its use would not infringe privately owned rights. Reference herein to any specific commercial product, process, or service by trade name, trademark, manufacturer, or otherwise does not necessarily constitute or imply its endorsement, recommendation, or favoring by the United States Government or any agency thereof. The views and opinions of authors expressed herein do not necessarily state or reflect those of the United States Government or any agency thereof.

\section{American Control Conference}

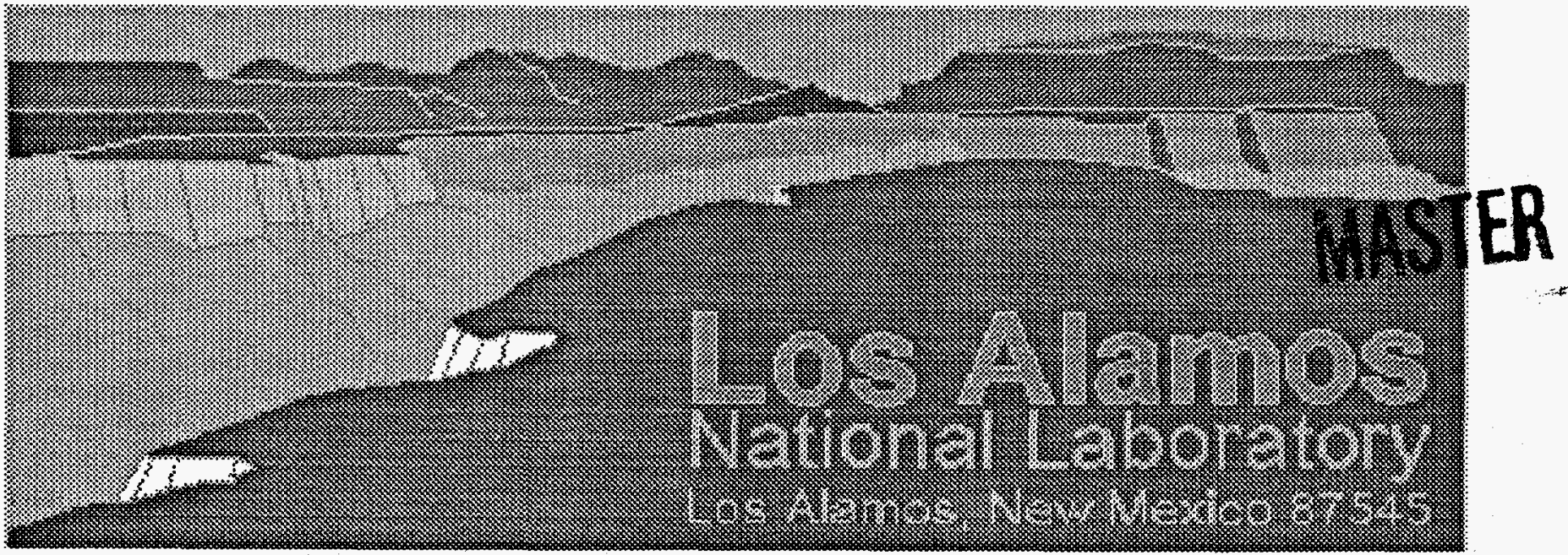

Los Alamos National Laboratory, an aftirmative actionequal opportunity employer, is operated by the University of California for the U.S. Department of Energy under contract W-7405-ENG-36. By acceptance of this article, the publisher recognizes that the U.S. Government retains a nonexclusive, royalty-free license to publish or reproduce the published form of this contribution, or to allow others to do so, for U.S. Govemment purposes. The Los Alamos National Laboratory requests that the publisher identify this article as work performed under the auspices of the U.S. Department of Energy. 


\section{DISCLAIMER}

Portions of this document may be illegible in electronic image products. Images are produced from the best available original document. 


\title{
A Two-Timescale Approach to Nonlinear Model Predictive Control
}

\author{
Kevin L. Buescher and Christopher C. Baum* \\ Los Alamos National Laboratory \\ MS F645 \\ Los Alamos, NM 87545 \\ klb@lanl.gov, baum@demos.lanl.gov
}

\begin{abstract}
Model Predictive Control (MPC) schemes generate controls by using a model to predict the plant's response to various control strategies. A problem arises when the underlying model is obtained by fitting a general nonlinear function, such as a neural network, to data: an exorbitant amount of data may be required to obtain accurate enough predictions. We describe a means of avoiding this problem that involves a simplified plant model which bases its predictions on averages of past control inputs. This model operates on a timescale slower than the rate at which the controls are updated and the plant outputs are sampled. Not only does this technique give better closed-loop performance from the same amount of open-loop data, but it requires far less on-line computation as well. We illustrate the usefulness of this two-timescale approach by applying it to a simulated exothermic continuously stirred tank reactor with jacket dynamics.
\end{abstract}

* Pleased address all correspondence to the first author. This work was performed under the auspices of the U.S. Department of Energy. 


\section{Introduction}

In Model Predictive Control (MPC), a model ${ }^{1}$ of the plant is used to predict the response of the plant to future control inputs. These predictions are incorporated into a finitehorizon optimization problem which is solved during each sampling interval in order to determine the best value for the next control input. This methodology is especially attractive for applications because constraints on the inputs can be explicitly accounted for and, when the model is nonlinear (e.g., a neural network), it can be readily applied to nonlinear plants.

There is a tradeoff in the choice of a sampling rate for MPC. If this rate is too low, the control system cannot react quickly to command changes and unmeasured disturbances. Further, a lower sampling rate may result in controls and responses that are less smooth, since there are fewer opportunities to change the control input. However, a number of problems arise if the rate is sufficiently high, and these problems are exacerbated by nonlinear models with many parameters, such as neural networks. A model for a fast sampling rate usually must depend on a large number of past values of plant inputs and outputs. The increased complexity of the model necessitates a large amount of data for adequate system identification. Further, a model for a faster sampling rate must be iterated more times to predict a given period of time into the future. This requires that the identification model give extremely accurate one-step predictions and greatly increases the computational burden of performing optimization during MPC.

In this paper, we describe a simple, but effective, means of circumventing the tradeoff described above. Our approach involves a model that operates on a timescale several times slower than the true sampling rate. This model averages the plant inputs over several sampling periods. Thus, the complexity of the model is greatly reduced for a given amount of past plant history fed into the model and far fewer iterations are needed for a given prediction horizon, but the control inputs are still updated at a fast rate.

We demonstrate the usefulness of this two-timescale approach by applying it to a simulated exothermic Continuously Stirred Tank Reactor (CSTR) with jacket dynamics. In this system, a reaction takes place inside a jacket immersed in a heat bath. The temperature of the heat bath is the controlled variable. The time lag in the control, and thus the difficulty of the control problem, can be adjusted by changing the heat transfer properties of the jacket. The unstable nature of this nonlinear plant and the time lag in the control make long prediction horizons with an accurate model necessary.

\footnotetext{
${ }^{1}$ Here we focus strictly on input-output models.
} 
The particular identification model we use here is based on a neural network called the Connectionist Nonlinear Local Spline (CNLS) network. This network is similar to a Radial Basis Function (RBF) network and provides a local linear approximation. The CNLS network is well-suited to on-line adaptation.

The rest of this paper is as follows. In the next section we discuss our two-timescale approach, which we call gapping, and explain how it addresses the problems described above. In Section 3, we describe the neural networks we use in the simulations and the associated identification procedures. We give details of our particular implementation of MPC in Section 4. In Section 5, we describe the lagged CSTR and present simulations which show how gapping can result in greatly improved control. We sum up our results and discuss how gapping has proven useful for plants other than the CSTR in Section 6 .

\section{Gapping: A Two-Timescale Approach .}

The conventional input-output model used for MPC relys on past values of $u$ and $y$ in order to predict the next value of $y$ :

$$
\hat{y}_{k+1}=\varphi\left(y_{k}, y_{k-1}, \ldots, u_{k}, u_{k-1}, \ldots\right) .
$$

For MPC, (2.1) must be iterated $N$ times in order to predict $y_{k+N}$.

As an alternative, we use a two-timescale approach in which the sampling of the plant outputs and the command changes still occur at the same fast rate, but the model uses averages of the plant's inputs over intervals of several sampling periods. We refer to this method as "gapping," and use the following structure for predictions.

$$
\hat{y}_{k+g}=\varphi\left(y_{k}, y_{k-g}, \ldots, \bar{u}_{k}, \bar{u}_{k-g}, \ldots\right),
$$

where

$k$ corresponds to the fast sampling rate,

$g$ is the integer sample "gap," and

$$
\bar{u}_{k}=\frac{u_{k}+u_{k+1}+\cdots+u_{k+g-1}}{g} .
$$

(Note that the conventional approach corresponds to $g=1$.) The underlying intuition is that a system such as the heat bath on the CSTR will tend to average its inputs over adjacent sampling periods when the sampling rate is high. 
For a given sequence of future control inputs, $y_{k+N}$ can be predicted by iterating (2.2) $N / g$ times. Thus, we can use the standard MPC machinery to find an optimal control input which we apply at the next time step. Note that this is done using a factor of $g$ fewer iterations (for a fixed prediction horizon) and model inputs (for a fixed interval of plant history), yet the controls are still updated every (fast) time step. This neatly addresses the problems described in the Introduction.

There is a concern that, by only predicting the output every $g$ 'th time step, the gapped method will result in oscillatory responses. We have not found this to be a problem. In fact, the gapped method helps avoid oscillations in the control which arise from the inability to predict far enough into the future. The results for the lagged CSTR demonstrate this quite graphically.

\section{System Identification}

In this section we give a brief description of the particular neural network model we use for system identification. We then discuss our off-line and on-line identification procedures.

\subsection{Network Structure}

The neural network model we use is the Connectionist Normalized Local Spline (CNLS) network developed by Jones $e t$ al. (see [1] and [2]). Its output is a normalized combination of basis functions. Each basis function, $\rho_{i}$, is a local function of the vector input $\vec{x}$ about a center $\vec{c}_{i}$ :

$$
\rho_{i}(\vec{x})=\exp \left[-\beta\left(\vec{x}-\vec{c}_{i}\right)^{\mathrm{T}}\left(\vec{x}-\vec{c}_{i}\right)\right],
$$

where the superscript $T$ indicates vector transpose. The quantity $\beta$ determines the "width" of the basis functions and will be held constant in this paper.

For a smooth function $f(\vec{x})$ and a set of centers $\vec{c}_{1}, \ldots, \vec{c}_{M}$, we have the identity

$$
f(\vec{x})=\frac{\sum_{j=1}^{M} f(\vec{x}) \rho_{j}(\vec{x})}{\sum_{k=1}^{M} \rho_{k}(\vec{x})} .
$$

Approximating $f(\vec{x})$ by the first two terms of its Taylor expansion about the centers yields a function of the form

$$
\varphi(\vec{x})=\sum_{j=1}^{M}\left[a_{j}+\left(\vec{x}-\vec{c}_{j}\right)^{\mathrm{T}} \vec{d}_{j}\right] \frac{\rho_{j}(\vec{x})}{\sum_{k=1}^{M} \rho_{k}(\vec{x})} .
$$

This structure is called a CNLS network with M nodes. The adjustable parameters are the centers, $\vec{c}_{i}$, the linear weights, $a_{i}$, and the gradient weights, $\vec{d}_{i}$. Note that if the 
gradient terms are discarded and the normalization (the division by the sum of the $\rho$ 's) is not performed, the CNLS network becomes a standard RBF network (see [3]).

\subsection{Off-line Identification}

The process of adjusting the network's parameters to approximate a dataset is known as training. We first train off-line using historical plant data. In our implementation, $a_{j}$ and $\vec{d}_{j}$ from Equation (3.3) above are the parameters which are adjusted during training. Because these parameters appear linearly in (3.3), there are simple and effective algorithms for adjusting them.

For input-output system identification, we train the network to map past plant inputs and outputs to the future output. The dataset is composed of $P$ points, $\left(\vec{x}^{p}, y^{p}\right)$, which are derived from historical plant data. Each $\vec{x}^{p}$ consists of past plant outputs and averaged inputs, $\left(y_{k}, y_{k-g}, \ldots, \bar{u}_{k}, \bar{u}_{k-g}, \ldots\right)$, and $y^{p}$ is the associated future output, $y_{k+g}$, as described in Section 2. The goal of the off-line training is to minimize the training error,

$$
\sum_{p=1}^{P}\left(y^{p}-\varphi\left(\vec{x}^{p}\right)\right)^{2}
$$

Before training commences, there are a number of non-adjustable quantities that must be selected: $M, \beta$, the number of past plant inputs and outputs in $\vec{x}$, and the centers $\vec{c}_{i}$. It is possible to adjust these parameters on-line, so that their initial values are not too important (see [4]) or to determine them by a cross-validation scheme involving independent test data. However, for simplicity, in this paper we chose the centers randomly from $\left\{x^{p}: p=1, \ldots, P\right\}$ and set the other fixed quantities by trial and error and prior experience.

We use a gradient descent scheme to approximately minimize (3.4), since this can also be used for on-line training. In order to succinctly describe the training algorithm, the network equation (3.3) will be rewritten in vector form as:

$$
\varphi(\vec{x})=\vec{w}^{\mathrm{T}} \vec{\tau}(\vec{x})
$$

using the following definitions.

$$
\vec{w}=\left(\begin{array}{c}
\vec{w}_{1} \\
\vec{w}_{2} \\
\vdots \\
\vec{w}_{M}
\end{array}\right), \text { with } \vec{w}_{j}=\left(\begin{array}{c}
a_{j} \\
\vec{d}_{j}
\end{array}\right), \text { and }
$$




$$
\vec{\tau}(\vec{x})=\left(\begin{array}{c}
\vec{\tau}_{1}(\vec{x}) \\
\vec{\tau}_{2}(\vec{x}) \\
\vdots \\
\vec{\tau}_{M}(\vec{x})
\end{array}\right), \text { with } \vec{\tau}_{j}(\vec{x})=\left(\begin{array}{c}
\rho_{j}(\vec{x}) \\
\left(\vec{x}-\vec{c}_{j}\right) \rho_{j}(\vec{x})
\end{array}\right) .
$$

We randomize the ordering of the training data and sweep through it repeatedly, adjusting the parameters for each training point in the sequence, $\left(\vec{x}^{i}, y^{i}\right)$, by

$$
\vec{w}_{i+1}=\vec{w}_{i}+\eta\left[y^{i}-\varphi\left(\vec{x}^{i}\right)\right] \frac{\vec{\tau}\left(\vec{x}^{i}\right)}{\vec{\tau}\left(\vec{x}^{i}\right)^{\mathrm{T}} \vec{\tau}\left(\vec{x}^{i}\right)}
$$

( $\vec{w}_{0}$ consists of small random numbers.) This update is essentially a form of least squares (see [1] and [2] for details). Since (3.4) is quadratic in the $a$ 's and $\vec{d}$ 's, there are none of the convergence problems associated with training feedforward sigmoidal networks.

When (3.4) or its change over a sweep through the data fall below a predetermined threshold, we halt the training. The quantity $\eta$ is the learning rate. Again, the halting criterion and learning rate could be chosen by cross-validation, but we have selected them based on prior experience in the simulations here.

\subsection{On-line Identification}

To supplement the off-line training, we also adapt the network parameters as on-line data becomes available. The on-line training is done by using (3.8) on each new data point, $\left(\vec{x}_{k}, y_{k}\right)$.

In addition to the slow adaptation that arises from using (3.8), we implement a "fast correction" scheme. This consists of adding the prediction error from the previous time step to the current prediction. In the simulations, this simple correction greatly improved the quality of the control. (However, in the presence of noise, this simplistic scheme would have to be altered to make it more robust.)

Training data acquired from a system under control tends to be quite correlated from time step to time step. This can lead to problems, as the network tends to overtrain in certain areas. We can deal with this by training on the time series which results from differencing the output of the plant. There are also more sophisticated methods for decorrelating the data (see [4], [5], and [6] for details and examples). This results in improved closed-loop performance, especially in situations where only a small amount of off-line data is available. However, we will stick with the simple on-line training described above to highlight the advantages of gapping. 


\section{Nonlinear MPC Details}

There are common elements to every MPC scheme (see [7], [8], and [9]). First, a model predicts the response of the plant to various control strategies. Second, a reference trajectory guides the plant (usually toward a setpoint). Third, a control strategy is determined by solving a finite-horizon optimization problem, and the initial part of this strategy is pursued until a new control is calculated. In this section, we give some of the details of the MPC scheme we use.

In the following, we describe the calculations performed between times $k-1$ and $k$ in order to determine $u(k)$. During this time, we consider $y(k)$ and our prediction of it, $\hat{y}(k)$, to be predetermined, since $u(k-1)$ is already being applied.

Suppose that we want to drive the plant to a setpoint, $y^{\circ}$. We take the reference trajectory, $y^{*}$, to be an exponential approach from the next (predicted) output, $\hat{y}(k)$, to $y^{\circ}$ :

$$
y_{k-1}^{*}(k+i g)=y^{\circ}-\left[y^{\circ}-\hat{y}(k)\right] \exp (-i \xi), i=0, \ldots
$$

(Note that the entire trajectory is updated at each time step, which we have emphasized by the notation $y_{k-1}^{*}$.) The rate of approach is controlled by $\xi$. If $\xi$ is small, the controller will try to slowly force the plant to reach $y^{\circ}$. If $\xi$ is very large, the controller will try to make the plant reach $y^{\circ}$ with every control move. Intermediate values of $\xi$ make the controlled response a bit sluggish, but we have found that this greatly reduces overshoots after a step change in $y^{\circ}$ and makes the control inputs much smoother in the presence of noise.

We restrict the control strategies to those for which the control remains constant after the first $h_{c}$ time steps; $h_{c}$ is the control horizon. Often, $h_{c}=1$ will be sufficient. The various strategies are evaluated by considering their effect on the next $h_{p}$ gapped output values; $h_{p}$ is the prediction horizon. We require that $h_{c} \leq h_{p}$.

For a Single Input, Single Output (SISO) plant, we determine a control strategy $\vec{u}$ by minimizing

$$
\begin{aligned}
J(\vec{u})= & \sum_{i=1}^{h_{p}} W^{y}\left(\hat{y}(k+i g)-y_{k-1}^{*}(k+i g)\right)^{2}+ \\
& \sum_{i=0}^{h_{c}-1} W^{u}(\bar{u}(k+i g)-\bar{u}(k+i g-g))^{2}
\end{aligned}
$$

over $\vec{u}=\left[\bar{u}(k), \ldots, \bar{u}\left(k+h_{c} g-g\right)\right]$. The first term of $J$ penalizes the difference between the predicted trajectory, $\hat{y}$, and the reference trajectory, $y_{k-1}^{*}$. The second term penalizes 
changes in the control inputs; this favors smooth controls. The weights $W^{y}$ and $W^{u}$ allow a trade-off between the relative penalties for the output errors and changes in inputs. For a non-SISO plant, the first and second terms are summed over the different outputs and inputs, respectively, and there are weights $W^{y}$ and $W^{u}$ for each output and input.

We minimize $J$ under constraints on the range of each input and on the allowed change in each input from one gapped time step to the next. We search for the minimum using a conjugate gradient technique and calculate the required gradients using an efficient recursive procedure. The minimization yields a sequence $\bar{u}^{*}(k), \ldots, \bar{u}^{*}\left(k+h_{c} g-g\right)$. At time $k$, we apply $\bar{u}^{*}(k)$, measure $y(k)$, and start the optimization process over again.

\section{Case Study: Exothermic CSTR}

The exothermic CSTR can pose difficult control problems, and the addition of jacket dynamics allows us to adjust the lag faced by the control variable. For these reasons, it provides an ideal testbed for comparing gapped and ungapped control schemes.

\subsection{Plant Description}

The plant that we study here is a modification of a model from [10] of an exothermic first-order reaction in a CSTR. This is a continuously fed process. The reaction takes place within a jacket that is immersed in a water bath. The state equations are as follows.

$$
\begin{gathered}
\frac{d x_{1}}{d t}=-x_{1}+D a\left(1-x_{1}\right) \exp \left(\frac{\gamma x_{2}}{\gamma+x_{2}}\right) \\
\frac{d x_{2}}{d t}=-x_{2}+B D a\left(1-x_{1}\right) \exp \left(\frac{\gamma x_{2}}{\gamma+x_{2}}\right)+\beta\left(x_{J}-x_{2}\right)+d \\
\frac{d x_{J}}{d t}=\delta\left(x_{J_{0}}-x_{J}\right) \\
y=x_{2}
\end{gathered}
$$

The state variable $x_{1}$ is the reactant concentration, $x_{2}$ is the dimensionless reactor temperature, and $x_{J}$ is the dimensionless jacket temperature. Equations (5.1) and (5.2) are from [10], and (5.3) has been added to model jacket dynamics. The control variable is $x_{J_{0}}$, which represents the temperature of the water bath (and the steady-state value of $x_{J}$ ). The parameter $\delta$ can be adjusted to alter the rate of heat transfer through the jacket. A disturbance in the feed temperature is modeled by $d$; the nominal value of $d$ is 0 . The values of the other parameters are $D a=0.072, \gamma=20.0, B=8.0$, and $\beta=0.3$. For an explanation of these quantities, see [10]. 


\subsection{Control Objective}

One feature of the exothermic CSTR which makes it difficult to control is the fact that it has unstable equilibrium points. Figure 1 shows the steady state operating curve for the CSTR. Points on the upper and lower segments of the curve, where $x_{J} \in[-1.0,0.5]$ and $x_{J} \in[-0.5,2.0]$, are stable. Points on the middle segment of the curve, where $x_{J} \in[-0.5,0.5]$, are unstable. Our objective will be to drive the system from a steady state on the lower segment to an unstable steady state on the middle segment. This is especially difficult when there is a lag in the control due to the jacket dynamics.

In steady state, the concentration, $x_{1}$, is a function of the reactor temperature, $x_{2}$. Since $x_{2}$ can be measured much more readily than $x_{1}$, we will take it as the output of the system and attempt to control it to setpoint.

A number of authors have reported results for controlling similar CSTR models ([11], [12], [13], and [14]). To our knowledge, none have combined the challenges of controlling from a stable setpoint to an unstable setpoint with a lag due to jacket dynamics. Both [11] and [12] control between setpoints on the lower leg of the steady state curve; [11] includes effects due to jacket dynamics. Both [13] and [14] deal with driving the system from a stable steady state to an unstable one, but there are no jacket dynamics. The difficulties one encounters when attempting to control the CSTR with linear techniques are explored in [14].

\subsection{Simulation Results}

We ran a number of simulations which demonstrate the advantages of using the gapped control, especially as the lag due to the jacket increases. In all of these simulations, the objective is to drive the system from the stable steady state $x_{2}=1.0$ to the unstable steady state $x_{2}=3.0$ starting at time 0 . Also, at time 50 , the system is subjected to an unmeasured step change of height 0.1 in the normalized feed temperature disturbance, $d$.

In all cases, the input structure to the neural network was three past values of plant output and three past values of control input. For the initial comparison between gapped and ungapped control, the sampling period was taken to be 0.5 (this corresponds to the time index $k$ ). Unless otherwise noted, the parameter values in the following table were used. 


\begin{tabular}{|c|c|c|}
\hline symbol & meaning & value \\
\hline \hline$M$ & number of nodes & 5 \\
\hline$\beta$ & smoothness factor for basis functions & 6.0 \\
\hline$\eta$ & learning rate & 0.1 \\
\hline$P$ & number of training patterns & 200 \\
\hline$h_{c}$ & control horizon & 1 \\
\hline$W^{y}$ & tracking error weight & 1.0 \\
\hline$W^{u}$ & control change weight & 0.3 \\
\hline $\max u$ & maximum input value & 1.3 \\
\hline $\min u$ & minimum input value & -1.0 \\
\hline $\max |\Delta u|$ & max control change per time step & 0.8 \\
\hline
\end{tabular}

The off-line training data was generated by subjecting the system to a long period of constant control inputs of random height and duration, followed by a short interval of the same type of input with a small amount of noise added to it. Figure 2 shows a sample of such training data. The solid line is the plant output and the diamonds are the control input. Note that there is very little data within the unstable region.

First, we give results for the ungapped control, where $g=1$. After some experimentation, we determined that the best control was achieved in this case by setting the prediction horizon to $h_{p}=6$ and the rate of approach for the reference trajectory to $\xi=0.1$. Longer prediction horizons would have been desirable, but the off-line training data was not sufficient to allow such accurate predictions. Figure 3 shows the response for essentially no lag in the jacket $(\delta=50)$ and Figure 4 shows the response for an intermediate amount of lag $(\delta=5)$. Note the oscillations in the response and the significant degradation in the response due to the increased lag.

For the gapped control, we found that a gap of $g=3$ with $h_{p}=4$ and $\xi=0.175$ gave satisfactory responses. Note that this corresponds to a prediction horizon of 12 on the timescale associated with the sampling rate. Figures 5 through 7 show the response under gapped control with jacket lags corresponding to $\delta=50,5$, and 2.5 , respectively. These responses are much smoother than those for the ungapped control, and the system recovers quickly from the added disturbance.

One may suspect that the poor response in the ungapped case is due to the sampling rate being too high. The last two simulations show that this is not the case. Here, we performed ungapped control, but changed the sampling period to 1.5 to match the gapped control above. We ran the training sequence for three times as long in order to give the same number of training points when the time series was sampled at the lower rate. After much experimentation, we arrived at $h_{p}=6$ and $\xi=0.025$ as good parameter 
values. This value of $\xi$ is quite low, but much larger values would not allow the controller to reach the setpoint. The responses, which are shown in Figures 8 and 9 for jacket lags corresponding to $\delta=50$ and 5 , are much worse than those for the gapped control.

\section{Concluding Remarks}

We have introduced a two-timescale method, "gapping," which is suited to solving some of the problems encountered when applying MPC with empirical, nonlinear models. We have shown that gapping gives greatly improved control for a simulated exothermic CSTR with jacket dynamics. This makes intuitive sense because this system exhibits two timescales; the cooling jacket tends to average out fluctuations in the temperature of the water bath. However, we have also found that gapping plays a key role in the control of other types of systems, most notably, distillation columns. We have applied it to a simulated methanol/ethanol distillation column (see [6] and [4]) and are in the process of applying it to a simulated and real linked tritium distillation system.

\section{References}

[1] R. D. Jones, Y. C. Lee, C. W. Barnes, G. W. Flake, K. Lee, P. S. Lewis, and S. Qian, "Function approximation and time series prediction with neural networks," Proceedings of the International Joint Conference on Neural Networks, San Diego, California, June 17-21, pp. I-649-666, 1990.

[2] R. D. Jones et al., "Nonlinear adaptive networks: A little theory, a few applications," Tech. Rep. LA-UR-91-273, Los Alamos National Laboratory, 1991.

[3] S. Haykin, Neural Networks: A Comprehensive Foundation. Ontario: MacMillan College Publishing Co., 1994.

[4] C. C. Baum, K. L. Buescher, and V. Hanagandi, "Adaptive nonlinear model predictive control of nonlinear chemical processes." Document in preparation.

[5] J. Shao, Y. C. Lee, and R. D. Jones, "Orthogonal projection method for fast online learning algorithm of radial basis function neural networks," in Proceedings of the World Conference on Neural Networks, vol. III, pp. 520-535, 1993. Also, Los Alamos report LA-UR-92-3292.

[6] C. C. Baum et al., "Adaptive chemical process control: DuPont/Los Alamos phase 1 report," Tech. Rep. LA-UR-94-1039, Los Alamos National Laboratory, 1994.

[7] C. E. Garcia, D. M. Prett, and M. Morari, "Model predictive control: Theory and practice-a survey," Automatica, vol. 25, no. 3, pp. 335-348, 1989. 
[8] D. Q. Mayne and H. Michalska, "Receding horizon control of nonlinear systems," IEEE Transactions on Automatic Control, vol. 35, no. 7, pp. 814-824, 1990.

[9] B. W. Bequette, "Nonlinear control of chemical processes: A review," Ind. Eng. Chem. Res., vol. 30, pp. 1391-1413, 1991.

[10] A. Uppal, W. H. Ray, and A. B. Poore, "On the dynamic behavior of continuous stirred tank reactors," Chemical Engineering Science, vol. 29, pp. 967-985, 1974.

[11] P. B. Sistu and B. W. Bequette, "Nonlinear predictive control of uncertain processes: Application to a CSTR," AIChE Journal, vol. 37, no. 11, pp. 1711-1723, 1991.

[12] M. J. Piovoso et al., "A comparison of three nonlinear controller designs applied to an adiabatic first-order exothermic reaction in a CSTR," in Proceedings of the 1992 American Control Conference, (San Diego, CA).

[13] E. Hernández and Y. Arkun, "Study of the control-relevant properties of backpropagation neural network models of nonlinear dynamical systems," Computers Chem. Engng., vol. 16, no. 4, pp. 227-240, 1992.

[14] V. Hanagandi, "Evaluation of linear control strategies for nonlinear plants," Tech. Rep. LA-UR-94-1029, Los Alamos National Laboratory, 1994. 


\section{Figure Captions}

1. Steady state operating curve for the CSTR: jacket temperature versus reactor temperature.

2. Sample training data: the thin solid line is the plant output and the diamonds are the control input.

3. Ungapped control with no lag in input $(\delta=50)$.

4. Ungapped control with intermediate lag in input $(\delta=5)$.

5. Gapped control $(g=3)$ with no lag in input $(\delta=50)$.

6. Gapped control $(g=3)$ with intermediate lag in input $(\delta=5)$.

7. Gapped control $(g=3)$ with substantial lag in input $(\delta=2.5)$.

8. Ungapped control with longer sampling period (3 times normal) and no lag in input $(\delta=50)$.

9. Ungapped control with longer sampling period (3 times normal) and intermediate lag in input $(\delta=5)$. 


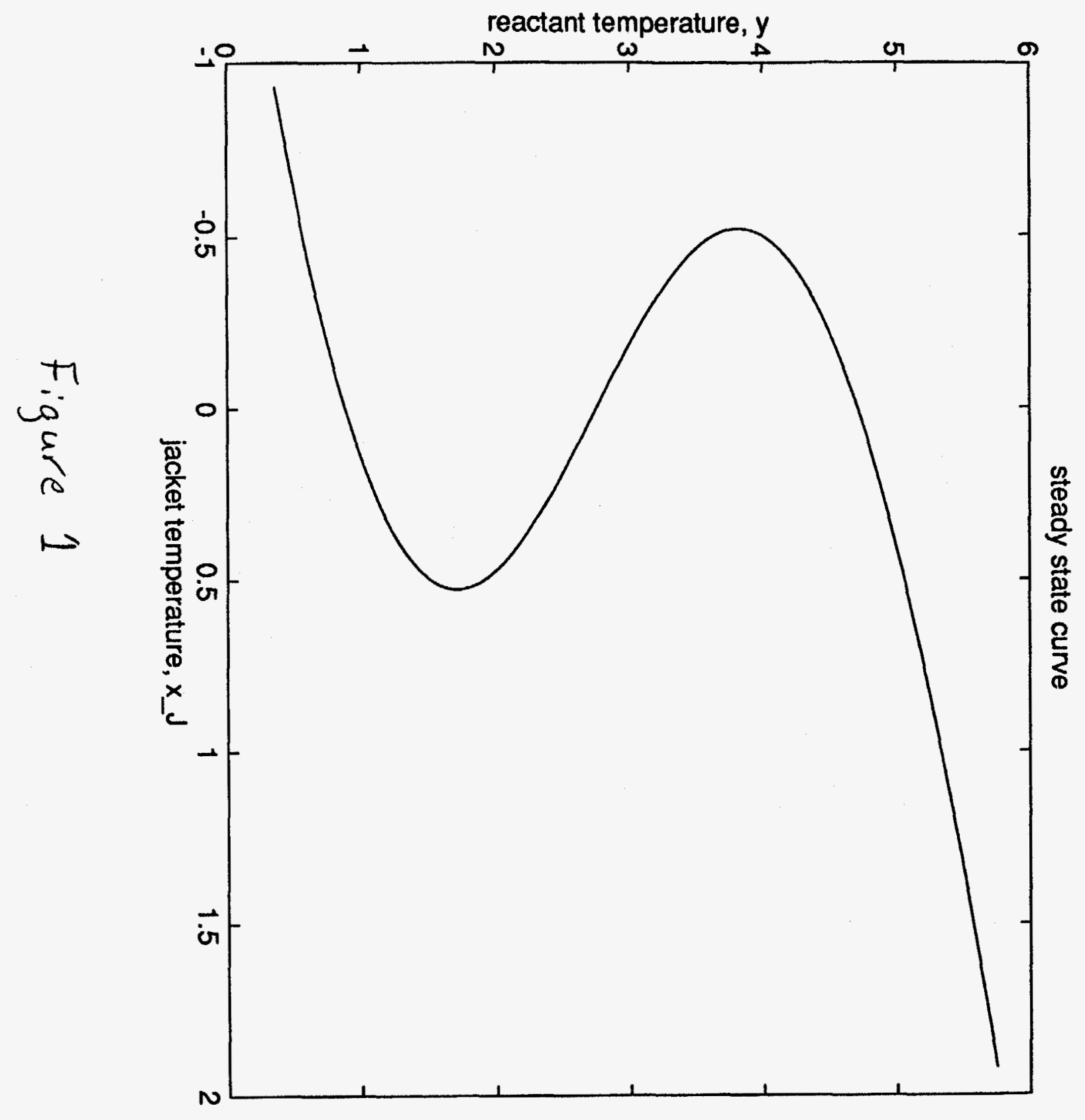


TRAINING SET FOR CONTROL NET

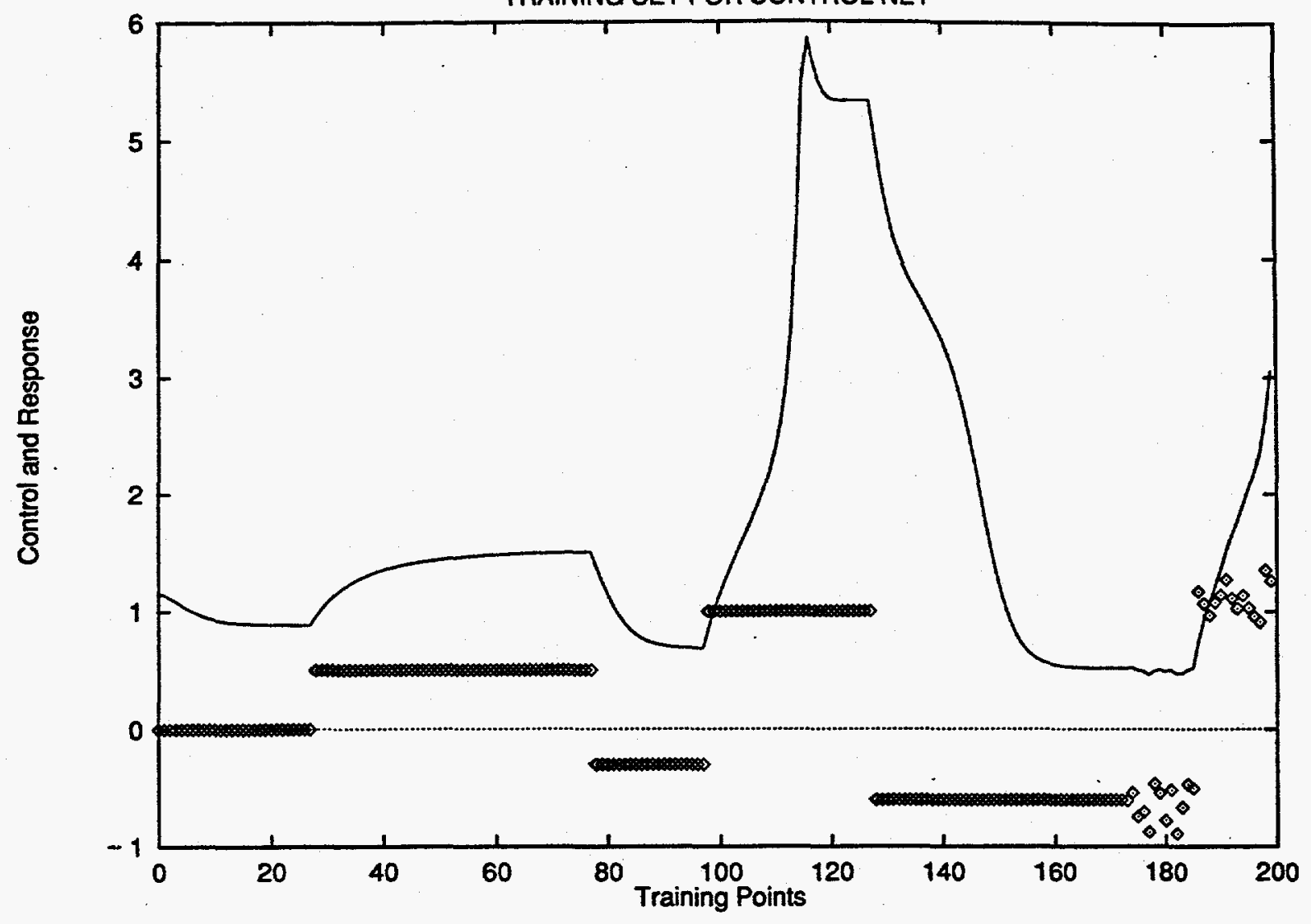

Figure 2

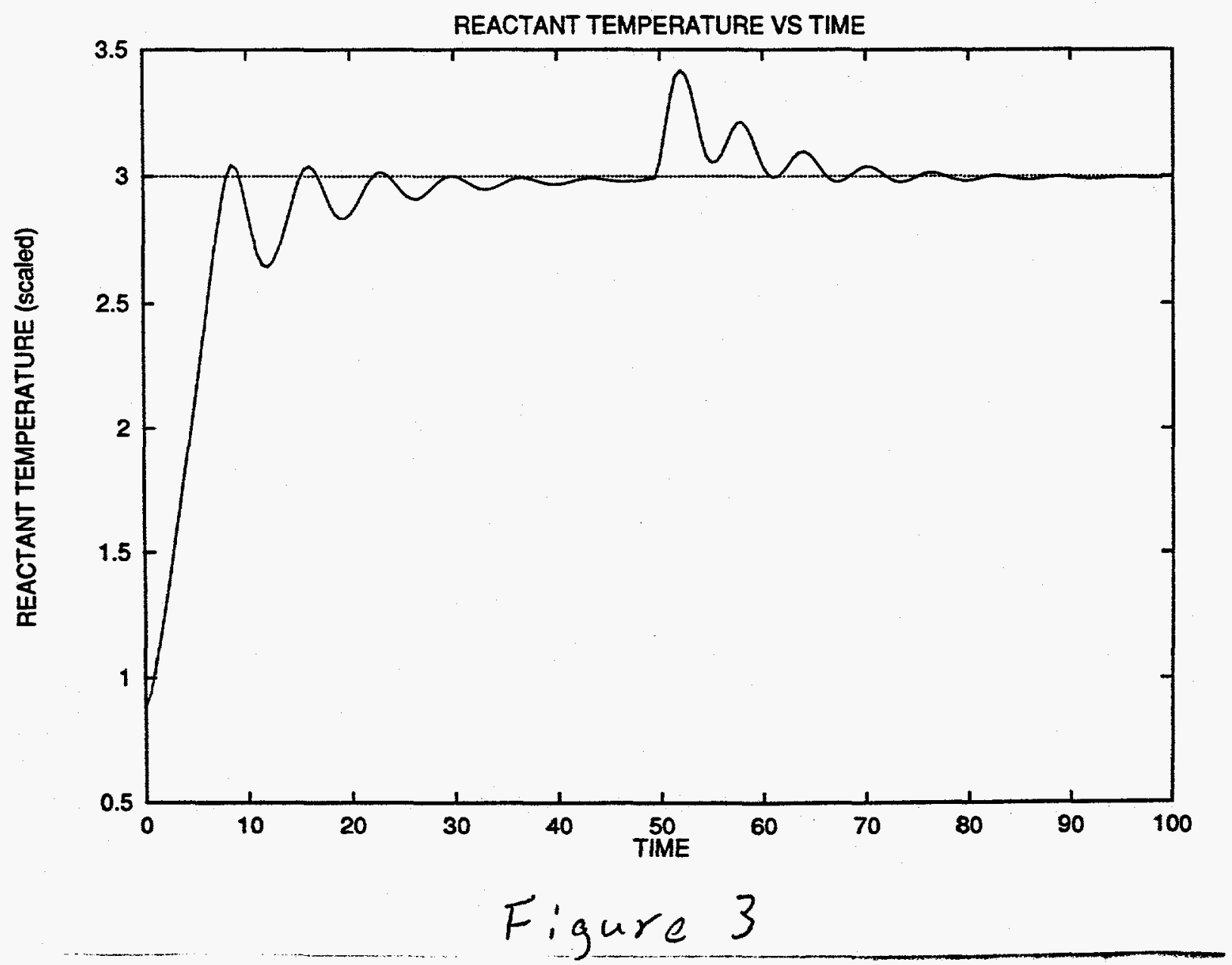


REACTANT TEMPERATURE VS TIME

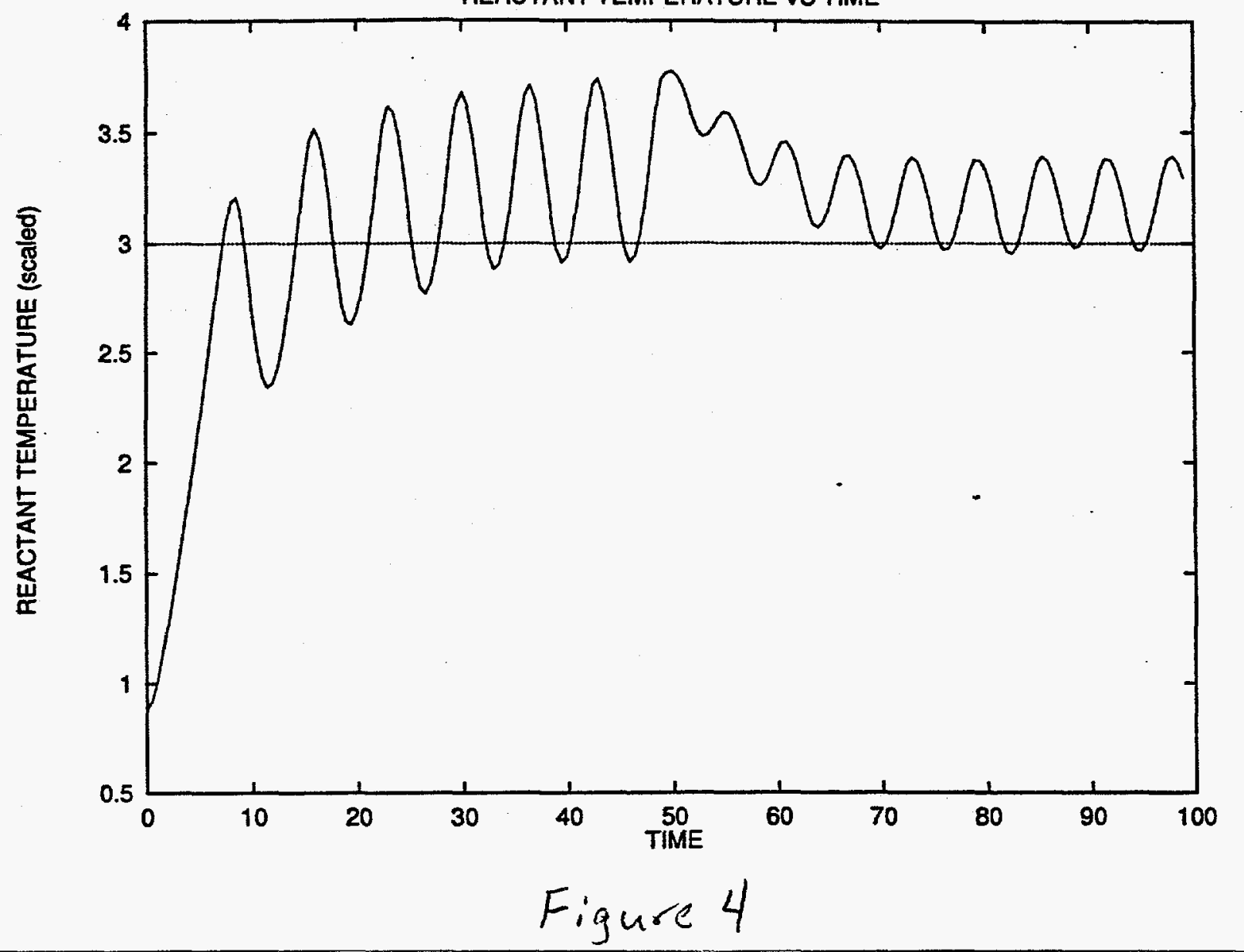

REACTANT TEMPERATURE VS TIME

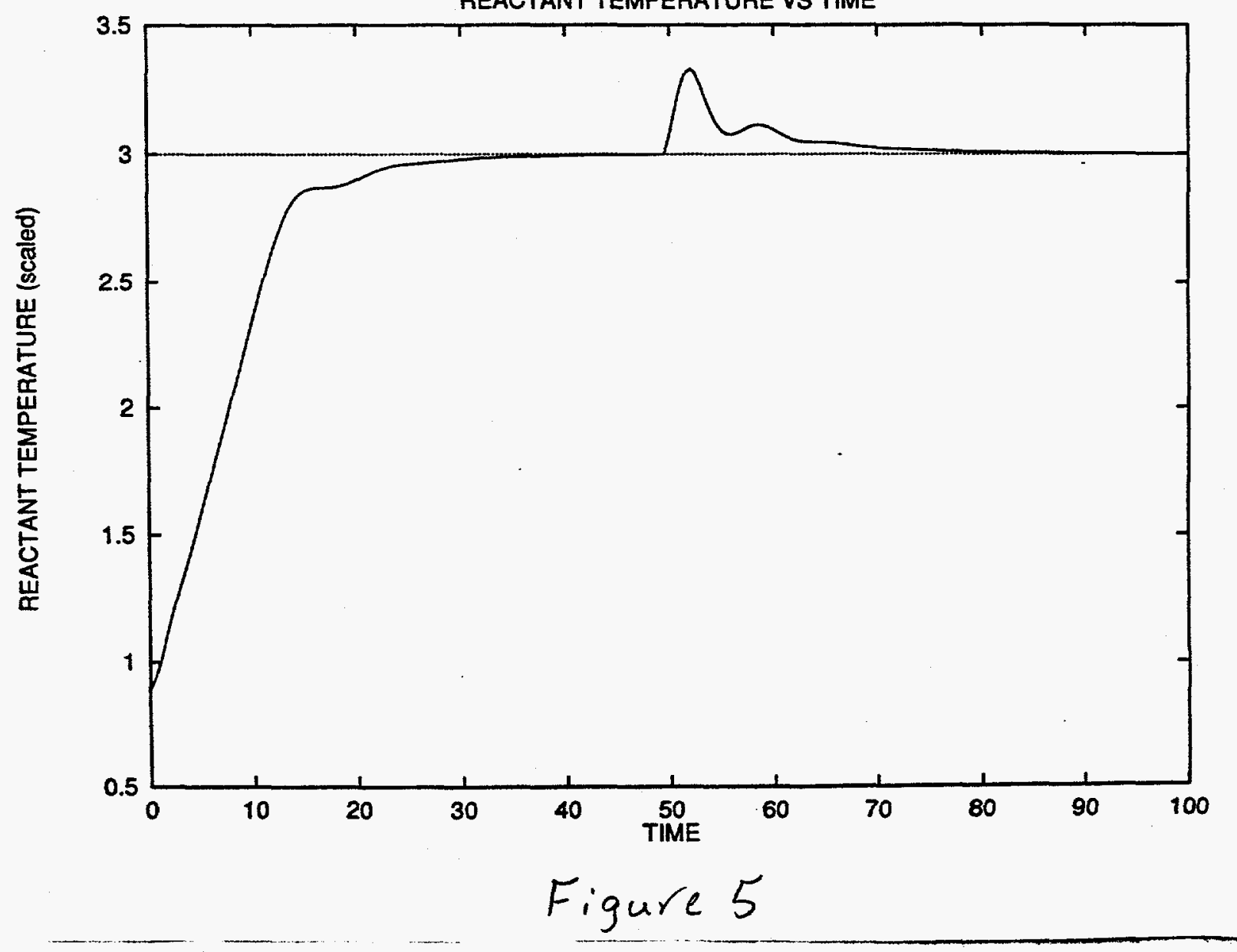


REACTANT TEMPERATURE (scaled)

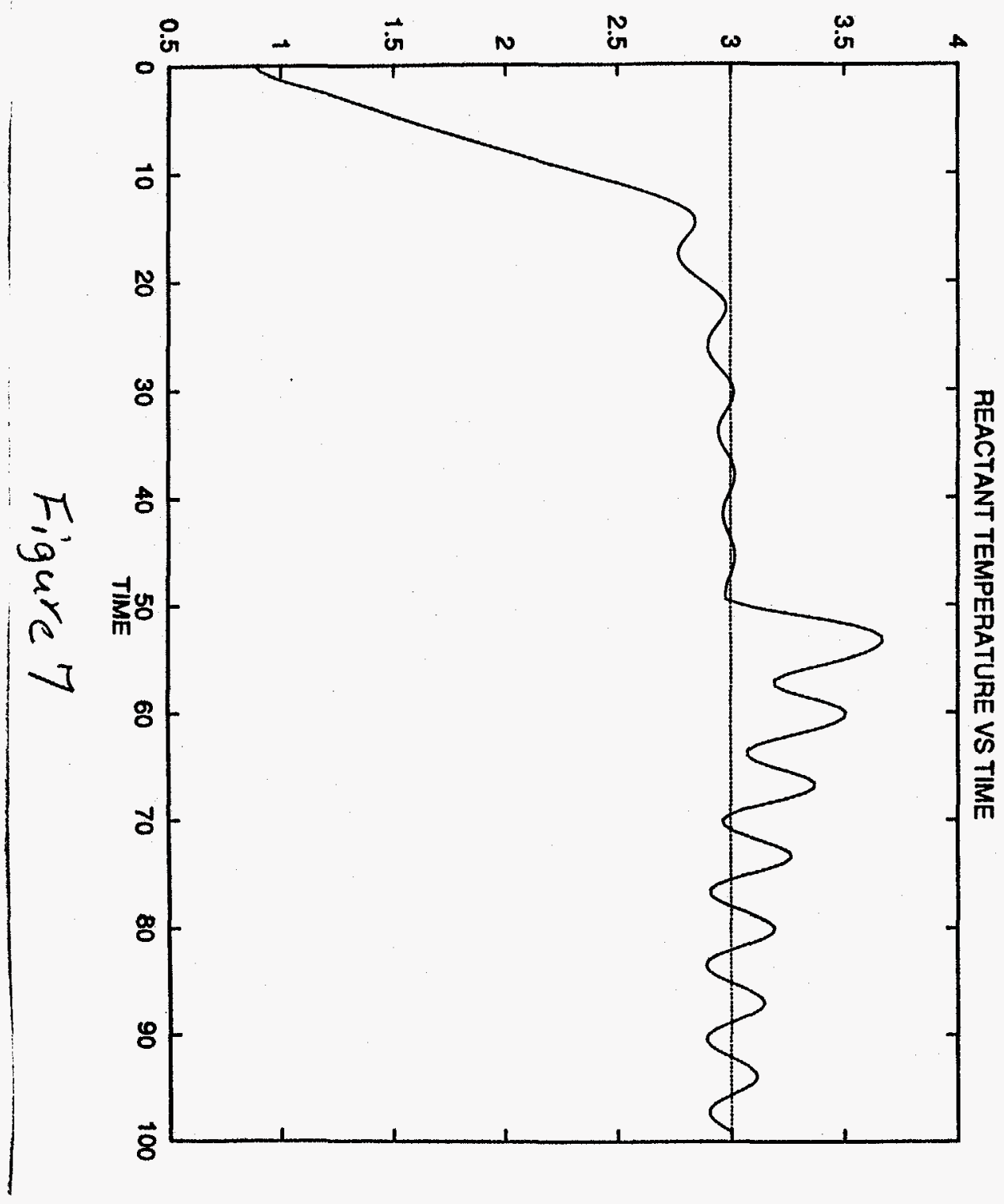

REACTANT TEMPERATURE (scaled)

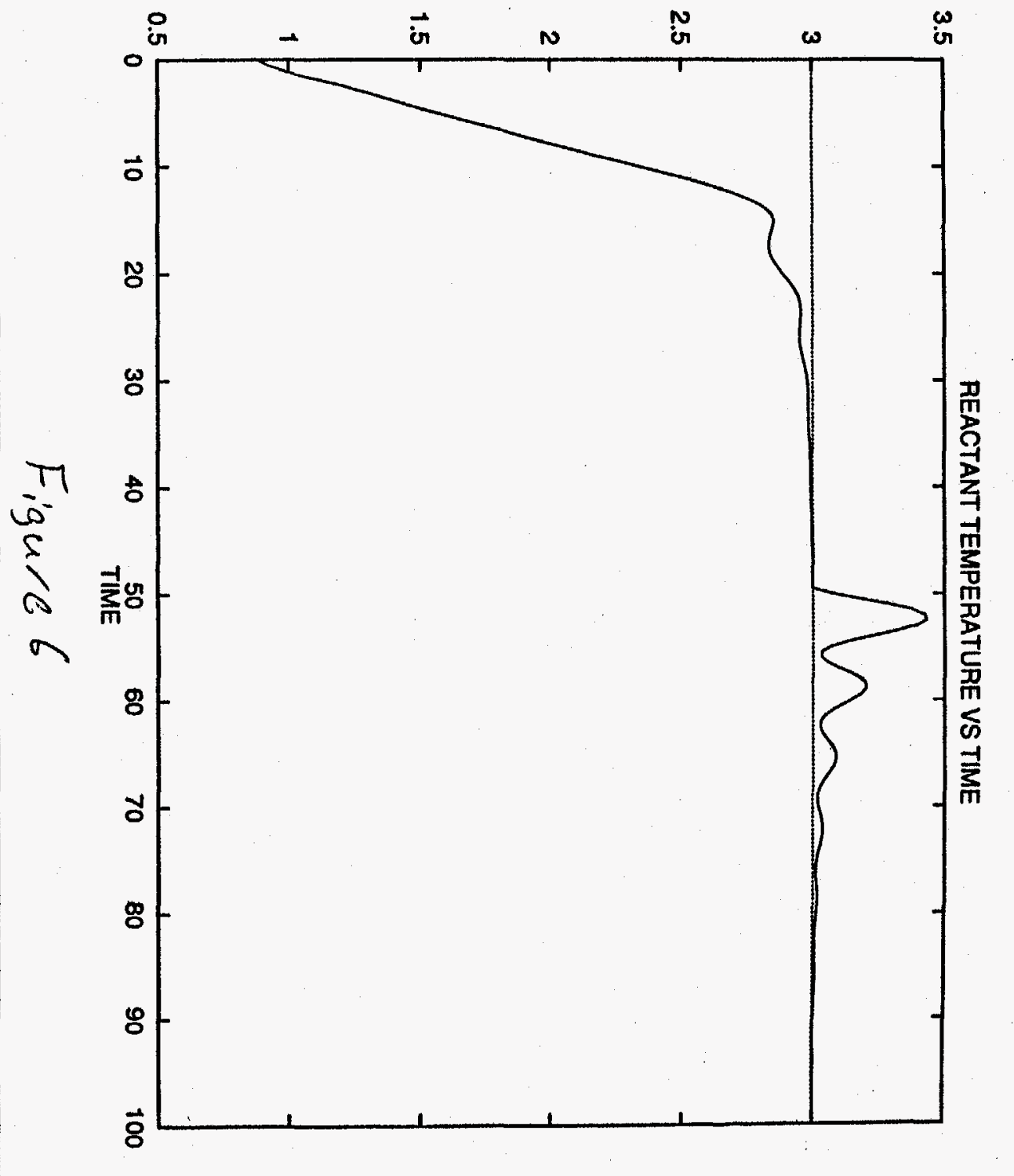


REACTANT TEMPERATURE VS TIME

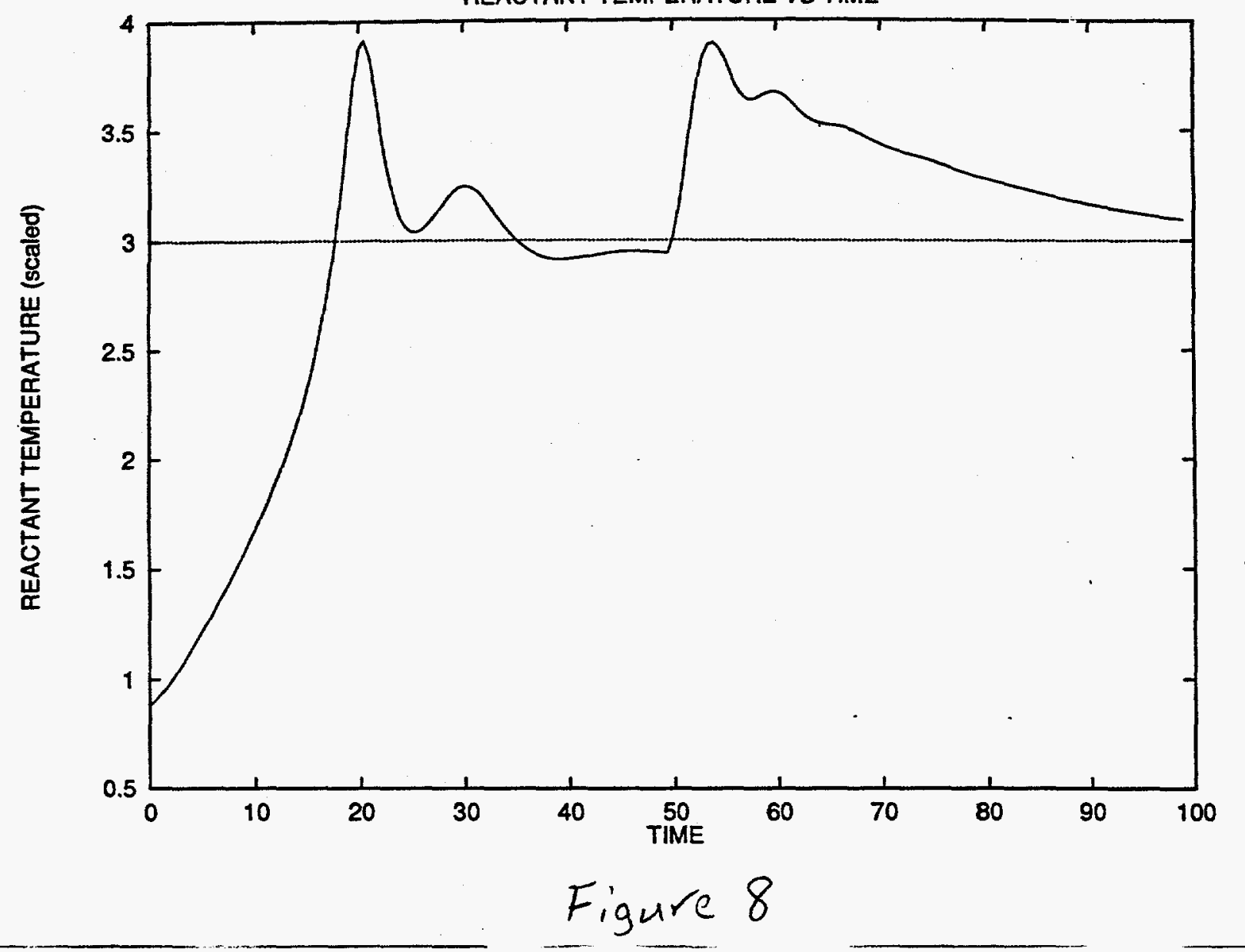

REACTANT TEMPERATURE VS TIME

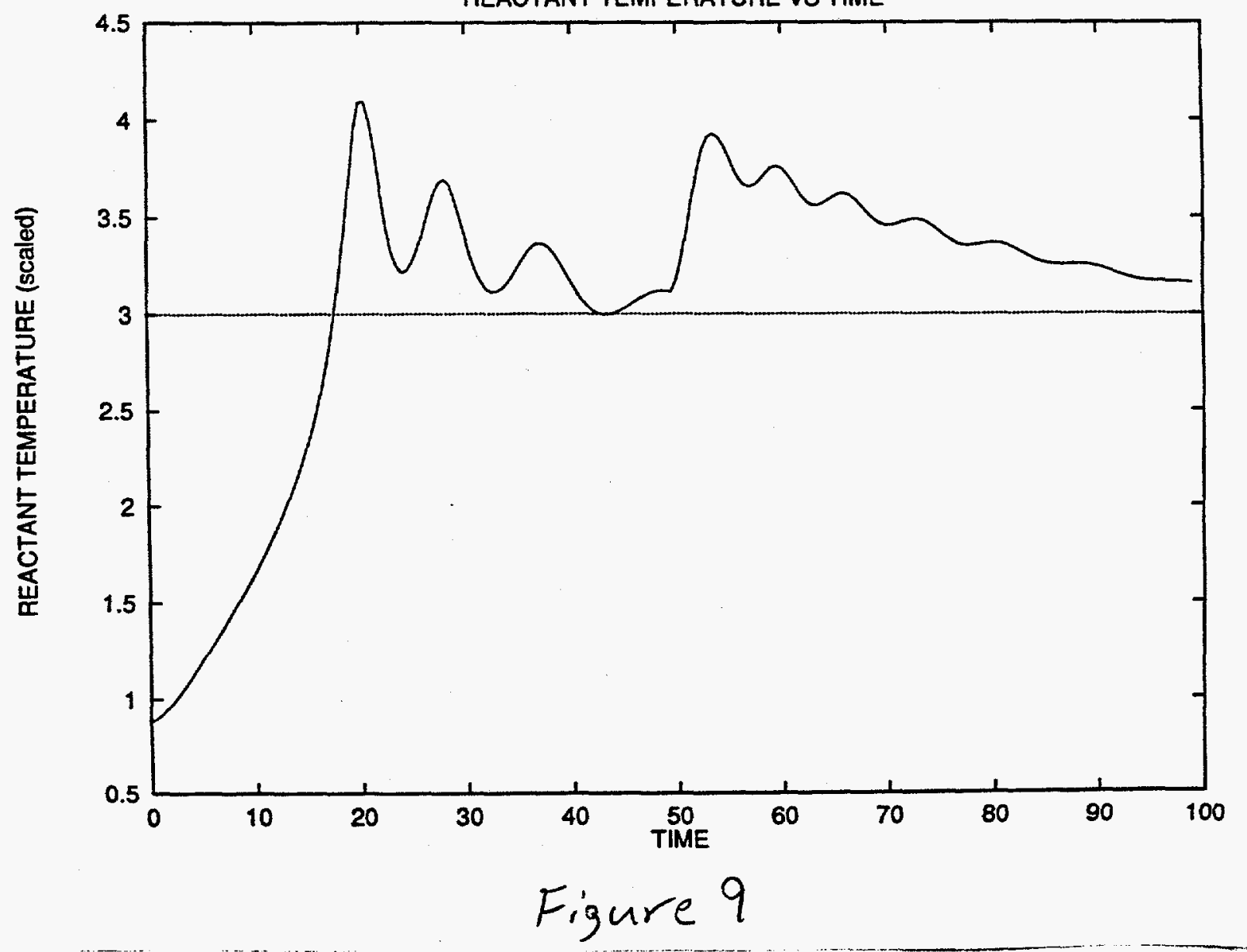

